\title{
Risk and resilience in trajectories of post-traumatic stress symptoms among first responders after the 2011 Great East Japan Earthquake: 7-year prospective cohort study
}

Taku Saito*, Florentine H. S. van der Does*, Masanori Nagamine, Nic J. van der Wee, Jun Shigemura, Taisuke Yamamoto, Yoshitomo Takahashi, Minori Koga, Hiroyuki Toda, Aihide Yoshino, Eric Vermetten and Erik J. Giltay

\section{Background}

First responders to disasters are at risk of developing posttraumatic stress disorder (PTSD). The trajectories of post-traumatic stress symptom severity differ among individuals, even if they are exposed to similar events. These trajectories have not yet been reported in non-Western first responders.

\section{Aims}

We aimed to explore post-traumatic stress symptom severity trajectories and their risk factors in first responders to the 2011 Great East Japan Earthquake (GEJE) - a historically large earthquake that resulted in a tsunami and a nuclear disaster.

\section{Method}

A total of 55632 Japan Ground Self-Defense Force (JGSDF) personnel dispatched to the GEJE were enrolled in this 7-year longitudinal cohort study. PTSD symptom severity was measured using the Impact of Event Scale-Revised. Trajectories were identified using latent growth mixture models (LGMM). Nine potential risk factors for the symptom severity trajectories were analysed using multinomial logistic regression.

\section{Results}

Five symptom severity trajectories were identified: 'resilient' (54.8\%), 'recovery' (24.6\%), 'incomplete recovery' (10.7\%),

'late-onset' (5.7\%), and 'chronic' (4.3\%). The main risk factors for the four non-resilient trajectories were older age, personal disaster experiences and working conditions. These working conditions included duties involving body recovery or radiation exposure risk, longer deployment length, later or no postdeployment leave and longer post-deployment overtime.

\section{Conclusions}

The majority of first responders to GEJE were resilient and developed few or no PTSD symptoms. A substantial minority experienced late-onset and chronic symptom severity trajectories. The identified risk factors can inform policies for prevention, early detection and intervention in individuals at risk of developing symptomatic trajectories.

\section{Keywords}

First responders; Great East Japan Earthquake; latent growth mixture models; post-traumatic stress symptoms; trajectory.

\section{Copyright and usage}

(c) The Author(s), 2022. Published by Cambridge University Press on behalf of the Royal College of Psychiatrists. This is an Open Access article, distributed under the terms of the creative Commons Attribution licence (https://creativecommons.org/ licenses/by/4.0/), which permits unrestricted re-use, distribution, and reproduction in any medium, provided the original work is properly cited.

\section{Background}

On 11 March 2011, northeastern Japan was hit by the largest earthquake in the country's recorded history. This earthquake caused the formation of a tsunami, which flooded a densely populated area and damaged the Fukushima Daiichi Nuclear Power Station, resulting in a release of radioactive material. This trifold disaster is known as the Great East Japan Earthquake (GEJE). In response to the GEJE, 107 000 first responders were dispatched, ${ }^{1}$ of which 70000 were Japan Ground Self-Defense Force (JGSDF) personnel. First responders to major disasters are typically exposed to many potentially traumatising experiences. As such, they are at an elevated risk of developing post-traumatic stress disorder (PTSD) compared with other first responders and the general population. ${ }^{2}$ Previous research has found high incidences of PTSD in first responders to natural disasters such as Hurricane Katrina, ${ }^{3}$ man-made mass violence such as the 2001 World Trade Center (WTC) attacks ${ }^{2}$ and nuclear events like the Chernobyl accident. ${ }^{4}$ In a non-Western cultural setting, this elevated risk was also found in first responders to the Wenchuan earthquake in China. ${ }^{5}$

Even if individuals are exposed to similar traumatic events, the development and course of PTSD symptoms vary. ${ }^{6}$ Identifying the

* Joint first authors. reasons for this heterogeneity and the risk factors for more adverse outcomes in various cultural settings is important to detect and prevent suffering caused by traumatic experiences in a professional setting. The current data-set contains longitudinal information on a large cohort of non-Western first responders to the GEJE - a complex and unprecedent disaster.

\section{Aims}

In two earlier studies on this cohort, we focused on the underlying risk factors for people with first-year probable PTSD and for probable PTSD after 6 months to 6 years of follow-up. ${ }^{7,8}$ In this study, we sought to identify PTSD symptom severity trajectories and the underlying risk factors in first responders after the GEJE.

Individuals with similar PTSD symptom severity trajectories can be identified by statistically modelling the clustering of symptom severity scores over time. Potential predictors of these trajectories can then be explored by comparing the characteristics of members of these different clusters. Latent growth mixture models (LGMM) use slopes and intercepts to identify multiple trajectories in the data-set. ${ }^{9}$ Commonly found symptom severity trajectories include 'resilient,' where few or no symptoms are experienced over the course of time; 'late-onset,' where symptoms start out relatively low but increase over time; 'recovery,' where symptoms 
start out high and taper off; and 'chronic,' where symptoms start out high and remain so over time. ${ }^{6}$ Only a few studies that use LGMM symptom trajectory analyses have been conducted among first responders, mostly with samples of first responders in the aftermath of the WTC attacks, ${ }^{10-13}$ which was a man-made disaster in a Western country. The number of natural disasters is increasing worldwide, ${ }^{14}$ so it is important to gain knowledge that can inform detection and prevention of long-term PTSD symptoms in first responders.

\section{Method}

\section{Study design and participants}

This study was part of a prospective longitudinal cohort study, the details of the design and participants have been described in prior research. $^{7,8}$ Of the 56753 individuals who participated in the initial study, we only included those with organisational service numbers and complete data on all predictor variables resulting in a sample of 55632 JGSDF personnel (98.0\% of the participants included in the initial study. See Supplementary Figure 1). Briefly, the first-year data were collected as part of mental health management measures for JGSDF personnel dispatched to the GEJE at 1, 6 and 12 months after mission completion. This was combined with data on PTSD symptoms obtained from the annual mental health surveys conducted from 2013 to 2018 (2-7 years after mission completion) at the end of each year over a period of approximately 2 months.

Written informed consent was not obtained and instead the study objectives and procedure were explained and an opportunity to refuse participation was provided. This study was conducted in accordance with the Declaration of Helsinki and the Strengthening the Reporting of Observational Studies in Epidemiology (STROBE) reporting guidelines. This study was approved by the Ethics Committee of the National Defense Medical College (Approval No. 2108).

\section{Psychological measures}

The characteristics of the psychological measures used are described in prior research. ${ }^{7,8}$ PTSD symptoms were investigated using the Japanese version of the Impact of Event Scale-Revised (IES-R), ${ }^{15,16}$ which has good test-retest reliability $(r=0.86, P<0.001)$ and high internal consistency (Cronbach's $\alpha=0.92-0.95$ ). ${ }^{15}$ The IES-R was completed at up to nine time points.

\section{Potential predictors}

We collected demographic information (gender, age), whether participants were personally affected by the disaster and work-related variables (deployment length, timing of post-deployment leave, risk of radiation exposure, post-deployment overwork, exposure to body recovery duties and rank).

\section{Statistical analyses}

A Cox regression analysis was used to determine risk factors for attrition. The distribution of baseline characteristics was assessed using Pearson's chi-squared tests. Because the distribution of IES-R scores was strongly positively skewed, sum scores were $\log _{\mathrm{e}}$-transformed. LGMMs were conducted to identify whether differential PTSD symptom severity trajectories were present in our data-set. We determined the optimal number of trajectories by starting with a model that grouped all participants into one trajectory and then adding one trajectory to each subsequent model until the optimum solution was identified. Initial values from the maximum likelihood estimates of a 1-class model were used for the multiple-trajectory models. The best fitting model was chosen using three fit indices: Akaike information criterion (AIC), Bayesian information criterion (BIC), and sample-size-adjusted BIC (SABIC). Entropy was used to assess the discriminatory power of the models. Best fit was determined by the lowest BIC, size-adjusted BIC, and AIC and entropy values approaching $1 .{ }^{17}$ As more classes are added, the AIC, BIC and SABIC tend to be lower. If this lowering after adding a class is small, the solution with the fewest classes should be accepted. We performed the LGMM with 1-7 latent trajectories and obtained the estimation process of these models. Chi-squared tests were used to compare the distribution of the potential predictors among the trajectory clusters.

After we conducted the LGMM, we used a multinomial logistic regression model to identify risk factors for the PTSD symptom severity trajectories, compared with the resilient trajectory. This yielded odds ratios (Ors) and 95\% CIs. To evaluate model fit for the regression, Nagelkerke's pseudo- $R^{2}$ was calculated. The LGMM and multinomial logistic regression were repeated including only participants for whom data were available on four or more measurement points, to ensure that the results were not greatly influenced by attrition. Also, the LGMM was repeated including a quadratic term, to assess whether this would result in a different distribution of participants over the trajectory clusters. All statistical analyses were performed using $\mathrm{R}$ (version 3.4 .3$)^{18}$ in Rstudio (R Foundation for Statistical Computing, Vienna, Austria, 2016. https://www.R-project.org/). We used the main packages 'nnet' for the multinomial log-linear models (version 7.3-12) and 'lcmm' for the latent class linear mixed models (version 1.8.1). The threshold for significance was defined as $P<0.05$.

\section{Results}

\section{Participants}

The demographic characteristics of the participants are summarised in Table 1. There was a total of 55632 participants (54 051 men, 97.2\%) included in the original study. Ages ranged from 18 to 63 years (mean 34.8, s.d. $=8.7$, median 34 ). There was high attrition throughout the 7-year measurement period (see Supplemental Figure 1, available at https://doi.org/10.1192/bjp.2022.2). Female JGDSF personnel $(z=13.92, P<0.001)$, those holding the lowest rank of private $(z=26.94, P<0.001)$ and those in the oldest cohort $(z=55.39, P<0.001)$ had the highest likelihood of attrition (see Supplementary Table 1).

\section{Major outcomes}

The estimation process of the LGMM analysis yielded fit indices and percentages of each latent trajectory over the 7-year period, which are summarised in Table 2. The five-trajectory solution was chosen because we have found that the AIC, BIC, loglik and SABIC improved up until the five-trajectory solution, after which these values stabilised. The entropy value rapidly declined after the five-trajectory solution (see Table 2 and Supplementary Figure 2). Additionally, in the six- and seven-trajectory solutions, some trajectories represented $0 \%$ of participants. The entropy value of the five-trajectory model $(0.81)$ indicated that a high proportion of participants was correctly classified.

Figure 1 shows the longitudinal trajectories of IES-R score. The majority of individuals followed a 'resilient' trajectory ( $n=30476$, $54.8 \%$ ), experiencing few or no symptoms over the course of monitoring. Additionally, we identified a 'recovery' trajectory $(n=13$ $658,24.6 \%)$ and an 'incomplete recovery' trajectory ( $n=5937$, $10.7 \%)$. For these trajectories, symptom severity tapered off over 
Table 1 Baseline sociodemographic variables in total participants $(n=55632)$ and each trajectory group

\begin{tabular}{|c|c|c|c|c|c|c|c|c|c|c|c|c|c|c|}
\hline & \multicolumn{2}{|c|}{ Total } & \multicolumn{2}{|c|}{ Resilient } & \multicolumn{2}{|c|}{ Recovery } & \multicolumn{2}{|c|}{$\begin{array}{l}\text { Incomplete } \\
\text { recovery }\end{array}$} & \multicolumn{2}{|c|}{ Late-onset } & \multicolumn{2}{|c|}{ Chronic } & \multirow[b]{2}{*}{$\chi^{2}$} & \multirow[b]{2}{*}{$P$} \\
\hline & $n$ & $\%$ & $n$ & $\%$ & $n$ & $\%$ & $n$ & $\%$ & $n$ & $\%$ & $n$ & $\%$ & & \\
\hline Participants & 55632 & 100.0 & 30476 & 54.8 & 13658 & 24.6 & 5937 & 10.7 & 3187 & 5.7 & 2374 & 4.3 & & \\
\hline Gender & & & & & & & & & & & & & 56.7 & $<0.001$ \\
\hline Men & 54051 & 97.2 & 29712 & 97.5 & 13209 & 96.7 & 5725 & 96.4 & 3127 & 98.1 & 2278 & 96.0 & & \\
\hline Women & 1581 & 2.8 & 764 & 2.5 & 449 & 3.3 & 212 & 3.6 & 60 & 1.9 & 96 & 4.0 & & \\
\hline Age, years & & & & & & & & & & & & & 1071.8 & $<0.001$ \\
\hline$\leq 25$ & 10016 & 18.0 & 5770 & 18.9 & 2766 & 20.3 & 878 & 14.8 & 349 & 11.0 & 253 & 10.7 & & \\
\hline $26-30$ & 10435 & 18.8 & 6315 & 20.7 & 2456 & 18.0 & 874 & 14.7 & 533 & 16.7 & 257 & 10.8 & & \\
\hline 31-35 & 9541 & 17.2 & 5502 & 18.1 & 2142 & 15.7 & 957 & 16.1 & 599 & 18.8 & 341 & 14.4 & & \\
\hline $36-40$ & 8144 & 14.6 & 4327 & 14.2 & 1834 & 13.4 & 933 & 15.7 & 601 & 18.9 & 449 & 18.9 & & \\
\hline $41-45$ & 7786 & 14.0 & 3870 & 12.7 & 1837 & 13.4 & 994 & 16.7 & 635 & 19.9 & 450 & 19.0 & & \\
\hline$\geq 46$ & 9710 & 17.5 & 4692 & 15.4 & 2623 & 19.2 & 1301 & 21.9 & 470 & 14.7 & 624 & 26.3 & & \\
\hline Rank & & & & & & & & & & & & & 322.6 & $<0.001$ \\
\hline Officer & 6269 & 11.3 & 3135 & 10.3 & 1537 & 11.3 & 801 & 13.5 & 410 & 12.9 & 386 & 16.3 & & \\
\hline Sergeant & 40788 & 73.3 & 22565 & 74.0 & 9699 & 71.0 & 4288 & 72.2 & 2508 & 78.7 & 1728 & 72.8 & & \\
\hline Private & 8575 & 15.4 & 4776 & 15.7 & 2422 & 17.7 & 848 & 14.3 & 269 & 8.4 & 260 & 11.0 & & \\
\hline Deployment length & & & & & & & & & & & & & 2637.6 & $<0.001$ \\
\hline$<1$ month & 23303 & 41.9 & 14870 & 48.8 & 4879 & 35.7 & 1681 & 28.3 & 1257 & 39.4 & 616 & 25.9 & & \\
\hline 1 to 3 months & 24710 & 44.4 & 12839 & 42.1 & 6587 & 48.2 & 2754 & 46.4 & 1519 & 47.7 & 1011 & 42.6 & & \\
\hline$\geq 3$ months & 7619 & 13.7 & 2767 & 9.1 & 2192 & 16.0 & 1502 & 25.3 & 411 & 12.9 & 747 & 31.5 & & \\
\hline Timing of post-deployment leave & & & & & & & & & & & & & 865.0 & $<0.001$ \\
\hline Early & 32130 & 57.8 & 18893 & 62.0 & 7545 & 55.2 & 2938 & 49.5 & 1790 & 56.2 & 964 & 40.6 & & \\
\hline Late & 20054 & 36.0 & 10114 & 33.2 & 5195 & 38.0 & 2448 & 41.2 & 1192 & 37.4 & 1105 & 46.5 & & \\
\hline None & 3448 & 6.2 & 1469 & 4.8 & 918 & 6.7 & 551 & 9.3 & 205 & 6.4 & 305 & 12.8 & & \\
\hline Post-deployment overwork & & & & & & & & & & & & & 870.3 & $<0.001$ \\
\hline Little to none & 37245 & 66.9 & 21723 & 71.3 & 8877 & 65.0 & 3407 & 57.4 & 2038 & 63.9 & 1200 & 50.5 & & \\
\hline$<3$ months & 13269 & 23.9 & 6428 & 21.1 & 3463 & 25.4 & 1751 & 29.5 & 837 & 26.3 & 790 & 33.3 & & \\
\hline$\geq 3$ months & 5118 & 9.2 & 2325 & 7.6 & 1318 & 9.7 & 779 & 13.1 & 312 & 9.8 & 384 & 16.2 & & \\
\hline Personal experience of the disaster & & & & & & & & & & & & & 1739.6 & $<0.001$ \\
\hline No & 50732 & 91.2 & 28863 & 94.7 & 12269 & 89.8 & 4894 & 82.4 & 2899 & 91.0 & 1807 & 76.1 & & \\
\hline Yes & 4900 & 8.8 & 1613 & 5.3 & 1389 & 10.2 & 1043 & 17.6 & 288 & 9.0 & 567 & 23.9 & & \\
\hline Body recovery duties & & & & & & & & & & & & & 1565.8 & $<0.001$ \\
\hline No & 39138 & 70.4 & 23449 & 76.9 & 8809 & 64.5 & 3403 & 57.3 & 2123 & 66.6 & 1354 & 57.0 & & \\
\hline Yes & 16494 & 29.6 & 7027 & 23.1 & 4849 & 35.5 & 2534 & 42.7 & 1064 & 33.4 & 1020 & 43.0 & & \\
\hline Duties with radiation exposure risk & & & & & & & & & & & & & 203.3 & $<0.001$ \\
\hline No & 46465 & 83.5 & 25961 & 85.2 & 11349 & 83.1 & 4687 & 78.9 & 2602 & 81.6 & 1866 & 78.6 & & \\
\hline Yes & 9167 & 16.5 & 4515 & 14.8 & 2309 & 16.9 & 1250 & 21.1 & 585 & 18.4 & 508 & 21.4 & & \\
\hline
\end{tabular}

time, starting out higher for the incomplete recovery trajectory than for the recovery trajectory. We also identified a 'late-onset' trajectory ( $n=$ $3187,5.7 \%)$, with symptom severity starting out low and increasing over time. Lastly, we identified a 'chronic' trajectory $(n=2374$, $4.3 \%)$, for which symptom severity was high throughout the study.

Figure 2 and Supplementary Table 2 show the ORs of analysed variables for each of the four symptomatic trajectories, compared with the resilient trajectory. Age $30-40$ years was a risk factor for the incomplete recovery, late-onset and chronic trajectories (OR $=1.44-3.45, P<0.001)$. Over 40 years of age was a strong universal risk factor for all symptomatic trajectories compared with the resilient trajectory $(\mathrm{OR}=1.21-4.45, P<0.001)$. Female gender was a risk factor for the recovery, incomplete recovery, and chronic trajectories $(\mathrm{OR}=1.60-2.58, P<0.001)$, but it was not significantly related to the late-onset trajectory $(\mathrm{OR}=0.99, P=0.930)$. Being personally affected by the disaster was a strong universal risk factor (OR $=1.60-3.69, P<0.001)$.

Furthermore, several deployment-related variables were universal risk factors; these included exposure to body recovery duties $(\mathrm{OR}=1.64-1.71, P<0.001)$, risk of radiation exposure $(\mathrm{OR}=$ $1.06-1.28, P \leq 03)$, longer deployment length $(\mathrm{OR}=1.27-3.47$, $P<0.001)$, later or no post-deployment leave $(\mathrm{OR}=1.16-1.96$, $P<0.01)$ and longer post-deployment overtime working (OR = $1.21-2.42, P \leq 0.01)$. Additionally, holding the lowest JGSDF rank of private was a risk factor for the recovery, incomplete recovery and chronic trajectories ( $\mathrm{OR}=1.21-1.90, P<0.001)$, but the opposite was observed for the late-onset trajectory $(\mathrm{OR}=0.69, P<0.001)$.

Ors for the analysed risk factors tended to be progressively higher in accordance with the symptom severity associated with the trajectories; Ors were typically low for the recovery trajectory, higher for the incomplete recovery trajectory and highest for the chronic trajectory. Although over time symptom severity increased for the late-onset trajectory, the Ors for the risk factors were typically lower for this trajectory.

As such, those following the symptomatic trajectories were significantly more likely to be older and to have experienced a higher intensity of work-related risk factors compared with those who were resilient. Individuals who followed the recovery, incomplete recovery and chronic trajectories were also more likely to be women and hold the rank of private. Individuals who followed a late-onset trajectory were significantly less likely to hold the rank of private than those who were resilient.

Nagelkerke's pseudo- $R^{2}$ for this regression was calculated at 0.12 . The results of the LGMM and multinomial logistic regression analysis stayed intact after repeating the analysis including only those participants who contributed data at four or more measurement time points (see Supplementary Figures 3 and 4). Including a quadratic term in the model did not greatly influence the distribution of participants over the trajectory clusters (see Supplementary Table 3). 
Our aim in this study was to identify trajectories of PTSD symptom severity in a large data-set of first responders to the 2011 GEJE. Also, we aimed to identify risk factors for symptomatic trajectories. Five discrete symptom severity trajectories were identified: resilient, recovery, incomplete recovery, late-onset and chronic. Approximately $10 \%$ of our participants experienced the most adverse late-onset or chronic symptom severity trajectories. We found that older age, personal disaster experiences, body recovery duties, risk of radiation exposure and prolonged working conditions were the most important risk factors for the four symptomatic trajectories. To the best of our knowledge, this is the first study to identify PTSD symptom severity trajectories and risk factors among first responders to the GEJE.

Our finding of five symptom severity trajectories was largely consistent with previous findings from LGMM studies on first responders to the WTC attacks, which reported between four and six trajectories. ${ }^{10-13}$ In our study, we identified the presence of two recovery trajectories; these other studies only found one. This difference might be because of our very large sample size, which made it possible to map symptom severity trajectories in more detail.

The risk factors for symptomatic trajectories identified in this study were largely consistent with the results from two previous studies on the current cohort, which examined the risk factors for probable PTSD manifested in the first year of follow-up ${ }^{7}$ and for the late-onset trajectory in up to 6 years of follow-up. ${ }^{8}$

\section{Older age}

Older age was a strong and consistent risk factor for all symptomatic trajectories. In previous literature, the effect of age at trauma exposure on the development of PTSD symptoms in adults has been mixed. In previous studies on first responders, older age has been found to be a risk factor for PTSD, ${ }^{19}$ and for symptomatic PTSD trajectories in WTC first responders. ${ }^{12}$ In other studies, however, age was not found to be a risk factor. ${ }^{20}$ Additionally, older studies on those tasked with identifying human remains ${ }^{21,22}$ found that lower age was a risk factor for more severe PTSD symptoms. Our results seem to support the findings of more recent literature on first responders. The elevated risk for older first responders could potentially be explained by a higher prevalence of prior workrelated traumatisation and longer-than-average length of service. ${ }^{23}$

\section{Being personally affected by the disaster}

Being personally affected by the disaster was a strong universal risk factor for the symptomatic PTSD symptom severity trajectories. Because the GEJE caused so much destruction, loss of life and injury in a wide area of northeastern Japan, a significant portion of the JGSDF first responders were directly and personally affected by the disaster and may have lost property or loved ones. Our study results are in line with previous literature that has consistently identified being personally affected by the potentially traumatic event as a risk factor. ${ }^{10,11,19}$

\section{Work-related risk factors}

We also identified several work-related risk factors for symptomatic trajectories. Body recovery duties, risk of radiation exposure, longer deployment length, later or no post-deployment leave and longer post-deployment overtime working were universal risk factors for the symptomatic trajectories. Several of these factors have been discussed in previous literature. Exposure to human remains was associated with symptomatic severity trajectories in WTC first responders. ${ }^{10,12}$ Risk of radiation exposure could exacerbate 


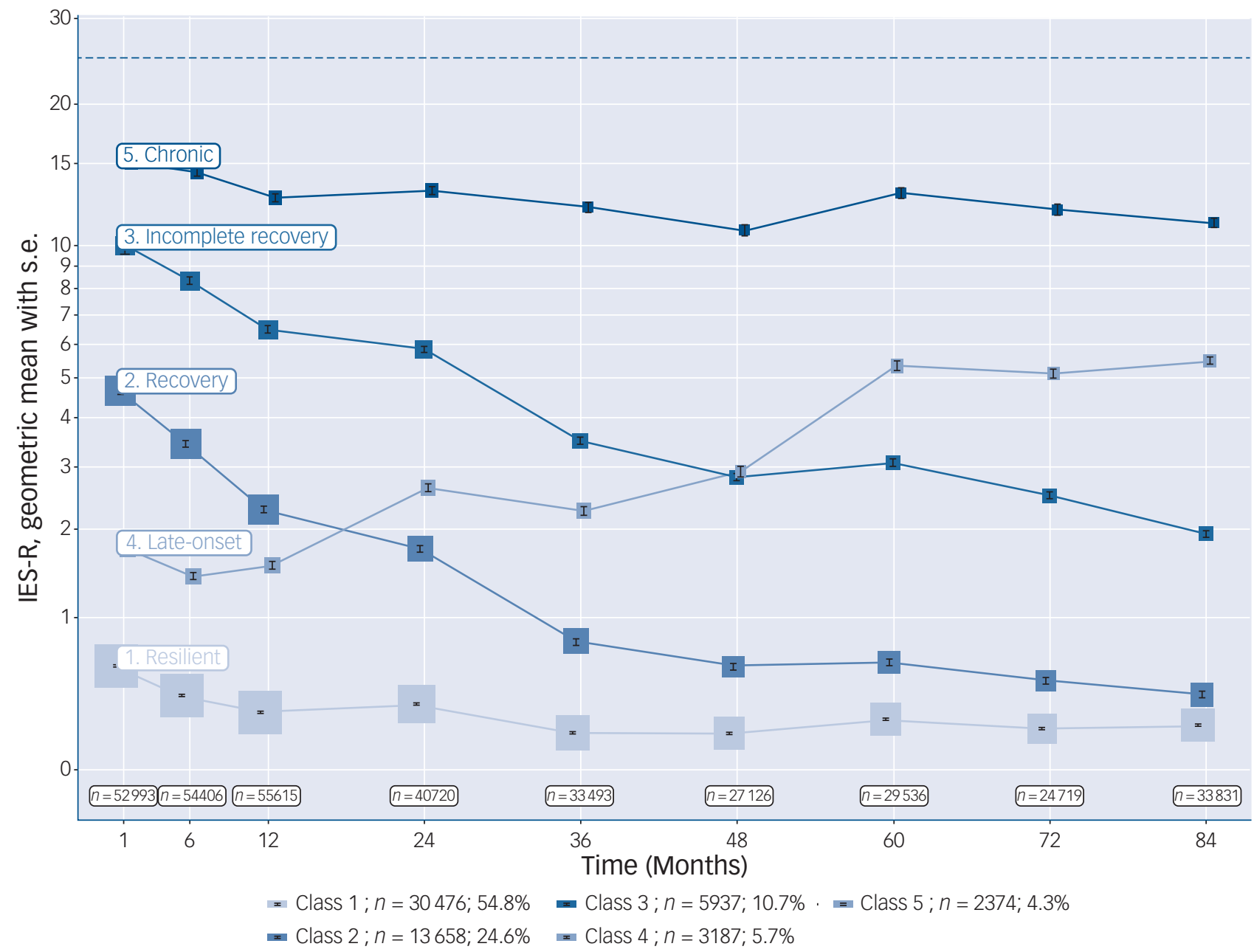

Fig. 1 Longitudinal trajectories for Impact of Event Scale-Revised (IES-R) scores. The error bars represent standard errors of the mean, and the size of each box is proportional to the number of subjects within that category at that time point. The dotted line represents the cut-off score for probable post-traumatic stress disorder (PTSD) in a Japanese sample.

Class 1, resilient; Class 2, recovery; Class 3, incomplete recovery; Class 4, late-onset'; Class 5, chronic.

psychological symptoms through physical health problems, neurological symptoms and worry about long-term consequences. ${ }^{4}$

Deployment length has also been previously identified as a risk factor, as longer duration of work at the WTC site increased the risk of a symptomatic trajectory. ${ }^{10-12}$ Other work-related risk factors that we have identified such as timing of post-deployment leave and post-deployment overtime working have not received much attention in previous LGMM studies. Knowing that these factors could have significant implications may be an important step towards preventing more severe longitudinal PTSD symptom severity because first responders with a large number of other risk factors could, for instance, be exempted from overtime working and be provided with post-deployment leave where possible.

\section{Rank}

First responders holding the lowest rank of private were more at risk of being on the recovery, incomplete recovery or chronic trajectories. To the best of our knowledge, lower rank has not been mentioned in previous literature as a risk factor for PTSD symptoms for first responders after a disaster, but it has been identified as a risk factor for work-related complaints and PTSD symptoms in military samples. ${ }^{24}$ Therefore, this finding could be a result of the military-style hierarchical structure of the JGSDF. Previous studies examining samples originating from Europe and the USA found that lower rank could increase the risk of symptomatic trajectories because of lower perceived control over the situation, lower perceived organisational support or perceived inadequate leadership. $^{23}$

\section{The late-onset trajectory}

Delayed onset of PTSD symptoms has previously been identified in many trauma populations, but the proportion of late-onset traumatic stress symptoms among people with PTSD is higher in professional samples. ${ }^{25}$ Interestingly, risk factors in our participants for the late-onset trajectory differed slightly from the other symptomatic trajectories. Female gender was a risk factor for the recovery, incomplete recovery or chronic trajectories, but not for the late-onset trajectory.

In previous literature, female gender has often been identified as a risk factor for a late-onset PTSD symptom severity trajectory in WTC first responders. ${ }^{10,12}$ In our data-set, the male-to-female ratio was approximately $1: 1$ in the late-onset trajectory, meaning that men were not at lower risk compared with women for developing this trajectory. Additionally, first responders holding the lowest 




Fig. 2 Risk factors for symptomatic trajectories, compared to the 'resilient' trajectory. Data are odds ratios with the error bars representing $95 \% \mathrm{Cl}$, and the size of each box is proportional to the number of participants. ${ }^{*} P<0.01, * * P<0.001$. 
rank of private were most at risk of being on the recovery, incomplete recovery or chronic trajectories, but those holding a higher rank were more at risk of developing a late-onset trajectory. This finding may in part be because of greater stigma around mental health and masculinity in Japan, compared with Western samples. This stigma could encourage men and those holding higher ranks to suppress initial symptoms, as they are often expected to have a strong characterand mental health problems are sometimes considered to reflect weakness. ${ }^{26}$ It is also possible that first responders holding higher ranks were exposed to more subsequent missions and associated traumatic stressors; privates are often temporary employees at the JGSDF. The presence of the late-onset trajectory in our data implies that first responders might develop serious problems as time goes on, even if initial symptom expression is low. These individuals may be overlooked without longitudinal follow-up. Therefore, it may be important to keep monitoring those who are exposed to risk factors but do not display high symptom severity.

\section{Limitations}

This data-set was collected as part of mental health monitoring and without the objectives of this study in mind. Therefore, data that could have provided more insight into the population were not collected, and known risk factors for the development of PTSD were not assessed. These include past exposure to disaster or traumatic events, past psychiatric history, immediate post-event traumatic symptoms, medical illness burden, post-deployment mental health support and social support.

Another limitation to our study is the low number of women included in the sample $(2.8 \%)$, which reflects the low number of women who were deployed to this mission. The total proportion of women in the JSDF was under $6 \%$ in 2011 , at the time of the GEJE. ${ }^{27}$ Also, there was substantial attrition throughout the years. Female JGSDF employees, those holding the lowest rank of private and those in the oldest cohort had the highest likelihood of attrition (see Supplementary Table 1). These results may be a reflection of the JGSDF system in which officers and sergeants have permanent employment, whereas privates are generally on fixed-term contracts. Furthermore, female SelfDefense Force personnel may have chosen to leave the service after marriage or childbirth because of the different social roles that were traditionally prescribed to men and women. This is further exacerbated by the rarity of higher-ranking female personnel, making women more likely to leave owing to a lack of career prospects. However, in recent years the JGSDF has been increasingly focused on recruiting and promoting women, and their prospects have been improving. ${ }^{27}$

Finally, symptoms may have been underreported because of the non-anonymous survey format, which may have led to concerns regarding stigma relating to mental health in a military setting. ${ }^{28}$

\section{Implications}

We identified five trajectories of PTSD symptom severity and analysed risk factors for the symptomatic severity trajectories in a 7-year cohort comprised of 55632 GSJSF first responders dispatched to the 2011 GEJE - an earthquake, tsunami and nuclear disaster. A substantial proportion of the first responders experienced late-onset and chronic PTSD severity trajectories. Risk factors for symptomatic trajectories included older age, being personally affected by the disaster and several deployment-related factors. For the response to future disasters, policymakers and healthcare providers should consider these risk factors to prevent and detect PTSD symptoms among first responders.
Taku Saito (D, MD, PhD, Department of Psychiatry, School of Medicine, Nationa Defense Medical College, Japan; Florentine H. S. van der Does (D), MSc, Department of Psychiatry, Leiden University Medical Center (LUMC), the Netherlands;

Masanori Nagamine, MD, PhD, Division of Behavioral Science, National Defense

Medical College Research Institute, Japan; Nic J. van der Wee, MD, PhD, Department of Psychiatry, Leiden University Medical Center (LUMC), the Netherlands; Jun Shigemura, MD, PhD, Department of Psychiatry, School of Medicine, National Defense Medical college, Japan; Taisuke Yamamoto, MD, PhD, Division of Behavioral Science, National Defense Medical College Research Institute, Japan; Yoshitomo Takahashi, MD, PhD, Division of Behavioral Science, National Defense Medical College Research Institute, Japan; Minori Koga, PhD, Department of Psychiatry, School of Medicine, National Defense Medical College, Japan; Hiroyuki Toda, MD, PhD, Department of Psychiatry, School of Medicine, National Defense Medical College, Japan; Aihide Yoshino, MD, PhD, Department of Psychiatry, School of Medicine, National Defense Medical College, Japan; Eric Vermetten (D), MD, PhD, Department of Psychiatry, Leiden University Medical Center (LUMC), the Netherlands and ARQ National Psychotrauma Center, the Netherlands; Erik J. Giltay, MD, PhD, Department of Psychiatry, Leiden University Medical Center (LUMC), the Netherlands

Correspondence: Masanori Nagamine. Email: nagaminemasanori@gmail.com

First received 26 Jul 2021, final revision 21 Dec 2021, accepted 5 Jan 2022

\section{Supplementary material}

To view supplementary material for this article, please visit https://doi.org/10.1192/bjp.2022.2.

\section{Data availability}

The data that support the findings of this study are available from the corresponding author, M.N., upon reasonable request.

\section{Author contributions}

T.S. and F.H.S.V.d.D. contributed equally as first authors. M.N. and E.J.G. had full access to all of the data in the study and take responsibility for the integrity of the data and the accuracy of the data analysis. Concept and design: M.N. and E.J.G. Acquisition, analysis, or interpretation of data: all authors. Drafting of the manuscript: T.S. and F.H.S.V.d.D., M.N., N.J.v.d.W., E.V. and E.J.G. Critical revision of the manuscript for important intellectual content: All authors. Statistical analysis: E.J.G., T.S. and F.H.S.V.d.D. Obtained funding: M.N. Administrative, technical, or material support: M.N., E.J.G. N.J.V.d.W., E.V. Supervision: M.N. and E.V.

\section{Funding}

This study was supported by the Japanese Society for the Promotion of Science KAKENHI (M.N., J.S. and A.Y., Grant Number 26461779) and a grant for Advanced Research on Military Medicine from the National Defense Medical College, Japan. The views expressed in this article are those of the authors and do not reflect the position or policy of Japan's National Defense Medical college or its Ministry of Defense

\section{Declaration of interest}

None.

\section{References}

1 Ranghieri F, Ishiwatari M. Learning from Megadisasters: Lessons from the Great East Japan Earthquake, World Bank Publications, 2014.

2 Wilson LC. A systematic review of probable posttraumatic stress disorder in first responders following man-made mass violence. Psychiatry Res 2015; 229: 21-6.

3 Osofsky HJ, Osofsky JD, Arey J, Kronenberg ME, Hansel T, Many M. Hurricane Katrina's first responders: the struggle to protect and serve in the aftermath of the disaster. Disaster Med Public Health Prep 2011; 5 (Suppl 2): S214-219.

4 Bromet EJ, Havenaar JM, Guey L. A 25 year retrospective review of the psychological consequences of the Chernobyl accident. Clin Oncol (R Coll Radiol) 2011; 23: 297-305.

5 Schenk EJ, Yuan J, Martel LD, Shi GQ, Han K, Gao X. Risk factors for long-term post-traumatic stress disorder among medical rescue workers appointed to the 2008 Wenchuan earthquake response in China. Disasters 2017; 41 788-802.

6 Bonanno GA, Mancini AD. Beyond resilience and PTSD: mapping the heterogeneity of responses to potential trauma. Psychol Trauma 2012; 4: 74.

7 Nagamine M, Yamamoto T, Shigemura J, Tanichi M, Yoshino A, Suzuki G, et al. The psychological impact of the great East Japan earthquake on Japan ground self-defense force personnel: a three-wave, one-year longitudinal study. Psychiatry 2018; 81: 288-96. 
8 Nagamine M, Giltay E, Shigemura J, van der Wee NJ, Yamamoto T, Takahashi Y, et al. Assessment of factors associated with long-term post-traumatic stress symptoms among first responders after the 2011 great East Japan earthquake: a 6-year prospective cohort study in 56388 first responders. JAMA Netw Open 2020; 3: e2018339.

9 Armour C, Shevlin M, Elklit A, Mroczek D. A latent growth mixture ovember approach to PTSD symptoms in rape victims. Traumatology (Tallahass Fla) 2012; 18: 20-8.

10 Feder A, Mota N, Salim R, Rodriguez J, Singh R, Schaffer J, et al. Risk, coping and PTSD symptom trajectories in world trade center responders. J Psychiatr Res 2016; 82: 68-79.

11 Maslow CB, Caramanica K, Welch AE, Stellman SD, Brackbill RM, Farfel MR. Trajectories of scores on a screening instrument for PTSD among world trade center rescue, recovery, and clean-up workers. J Trauma Stress 2015; 28: 198-205.

12 Pietrzak RH, Feder A, Singh R, Schechter CB, Bromet EJ, Katz CL, et al. Trajectories of PTSD risk and resilience in world trade center responders: an 8year prospective cohort study. Psychol Med 2014; 44: 205-19.

13 Bowler RM, Harris M, Li J, Gocheva V, Stellman SD, Wilson K, et al. Longitudinal mental health impact among police responders to the 9/11 terrorist attack. Am J Ind Med 2012; 55: 297-312.

14 Centre for Research on the Epidemiology of Disasters. Human Cost of Disasters: An Overview of the Last 20 Years. UN Office for Disaster Risk Reduction, 2020.

15 Asukai N, Kato H, Kawamura N, Kim Y, Yamamoto K, Kishimoto J, et al. Reliability and validity of the Japanese-language version of the impact of event scale-revised (IES-R-J): four studies of different traumatic events. J Nerv Ment Dis 2002; 190: 175-82.

16 Weiss DS. The Impact of Event Scale-Revised. In Assessing Psychological Trauma and PTSD ( $2^{\text {nd }}$ Edition) (ed JP Wilson, TM Keane): 168-89. The Guilford Press, 2004.

17 Jung T, Wickrama KA. An introduction to latent class growth analysis and growth mixture ovember. Soc Personal Psychol Compass 2008; 2: 302-17.

$18 R$ : A language and environment for statistical computing. $R$ Foundation for Statistical Computing. R, 2019 (https://www.R-project.org/).

19 Pietrzak RH, Schechter CB, Bromet EJ, Katz CL, Reissman DB, Ozbay F, et al. The burden of full and subsyndromal posttraumatic stress disorder among police involved in the world trade center rescue and recovery effort. J Psychiatr Res 2012; 46: 835-42.

20 Motreff Y, Baubet T, Pirard P, Rabet G, Petitclerc M, Stene LE, et al. Factors associated with PTSD and partial PTSD among first responders following the Paris terror attacks in ovember 2015. J Psychiatr Res 2020; 121: 143-50.

21 Jones DR. Secondary disaster victims: the emotional effects of recovering and identifying human remains. Am J Psychiatry 1985; 142: 303-07.

22 McCarroll JE, Fullerton CS, Ursano RJ, Hermsen JM. Posttraumatic stress symptoms following forensic dental identification: Mt. Carmel, Waco, Texas. Am J Psychiatry 1996; 153: 778-82.

23 Brooks SK, Dunn R, Sage CA, Amlôt R, Greenberg N, Rubin GJ. Risk and resilience factors affecting the psychological wellbeing of individuals deployed in humanitarian relief roles after a disaster. J Ment Health 2015; 24(6): 385-413.

24 Rona RJ, Hooper R, Jones M, Iversen AC, Hull L, Murphy D, et al. The contribution of prior psychological symptoms and combat exposure to post Iraq deployment mental health in the UK military. J Trauma Stress 2009; 22: 11-9.

25 Utzon-Frank N, Breinegaard N, Bertelsen M, Borritz M, Eller NH, Nordentoft M, et al. Occurrence of delayed-onset post-traumatic stress disorder: a systematic review and meta-analysis of prospective studies. Scand J Work Environ Health 2014; 40: 215-29.

26 Chan RK, Hayashi K. Gender roles and help-seeking behaviour: promoting professional help among Japanese men. J Soc Work (Lond) 2010; 10: 243-62.

27 Nakagawa M. Gaps between systems and reality in the Japan ground selfdefense force: what the leadership should learn for gender integration. Disaster Divers Emerg Prep 2019; 146: 101.

28 McLay RN, Deal WE, Murphy JA, Center KB, Kolkow TT, Grieger TA. On-the-record screenings versus anonymous surveys in reporting PTSD. Am J Psychiatry 2008; 165: 775-6. 